\title{
Effects of hypoxic preconditioning combined with altitude training on CD55, CD59 and the immune function of swimmers
}

\author{
Xi Wang ${ }^{1,2}$, Lin Huang ${ }^{3}$, Huan Gao ${ }^{1,2}$ \\ ${ }^{1}$ Laboratory of Hypoxia, Shanghai Research Institute of Sports Science, Shanghai, China; ${ }^{2}$ Shanghai Anti-doping Agency, Shanghai, China; \\ ${ }^{3}$ Department of Physical Education, Shanghai Dianji University, Shanghai, China \\ Contributions: (I) Conception and design: X Wang; (II) Administrative support: H Gao; (III) Provision of study materials or patients: X Wang, H \\ Gao; (IV) Collection and assembly of data: X Wang, L Huang; (V) Data analysis and interpretation: L Huang, X Wang; (VI) Manuscript writing: All \\ authors; (VII) Final approval of manuscript: All authors. \\ Correspondence to: Lin Huang, PhD. Department of Physical Education, Shanghai Dianji University, No. 300 Shuihua Road, Pudong New District, \\ Shanghai, China. Email: huang_229@126.com.
}

Background Hypoxic preconditioning refers to a certain intensity and time of hypoxic exposure before hypoxic stress, which makes the body produce endogenous protection to enhance the body's tolerance to subsequent more severe hypoxia. However, there are few studies on the effects of hypoxic preconditioning combined with altitude training on the immune system of athletes.

Methods: Nine swimmers from Shanghai underwent 3-week hypoxic preconditioning [living high-training low (HiLo)] combined with 3-week altitude training. CD55 and CD59 expression in red blood cells (RBCs), CD55 and CD59 expression in white blood cells (WBCs), RBC count, WBC count, T lymphocyte CD3, CD4, CD8 expression, and immunoglobulins IgG, IgM, and IgA were measured 4 times: before the start of hypoxic preconditioning, in the first week of hypoxic preconditioning, at the end of hypoxic preconditioning (i.e., before the start of altitude training), and at the end of altitude training.

Results: CD55 and CD59 expression in RBCs significantly increased in the first week of hypoxic preconditioning $(\mathrm{P}<0.05)$, returned to baseline levels at the end of preconditioning, and significantly increased again during altitude training $(\mathrm{P}<0.05)$. CD55 and CD59 expression in WBCs decreased significantly during hypoxic preconditioning $(\mathrm{P}<0.05)$ and increased significantly during altitude training $(\mathrm{P}<0.05)$. CD3 expression first decreased and then increased in the hypoxic preconditioning phase, then decreased again in the altitude training phase. However, there was no significant difference in each phase. CD4/CD8 expression after altitude training was significantly lower than that before altitude training $(\mathrm{P}<0.05)$, but was not significantly different from that before the start of hypoxic preconditioning. IgG, $\operatorname{IgM}$, and IgA did not fluctuate significantly throughout the experimental phase.

Conclusions: After hypoxic preconditioning combined with altitude training, the expression of CD55 and CD59 on the surface of RBCs and WBCs increased significantly, and T lymphocyte CD4/CD8 expression also increased. These results suggest an improvement in the complement regulation system and RBC immune function. Hypoxic preconditioning can therefore improve immunity and enhance the physical function of athletes during altitude training.

Keywords: Hypoxic preconditioning; altitude training; swimmers; immune function

Submitted Jul 13, 2020. Accepted for publication Jan 17, 2021.

doi: 10.21037/apm-20-2379

View this article at: http://dx.doi.org/10.21037/apm-20-2379 


\section{Introduction}

Altitude training is an important training method to improve ability in physical endurance sports (1). It can dilate vessels, thicken blood vessels and blood vessel walls, and increase blood volume substantially, thus improving the cardiovascular system of athletes. Altitude training enhances the capacity to tolerate lactic acid by increasing maximal oxygen uptake and hemoglobin concentration. It has been shown that altitude training 3-4 per week can improve swimming ability in elite swimmers, and the improvement can be maintained for a considerable period of time after the end of altitude training (2). Altitude exposure is a type of physiological stress, and training at high altitude will exacerbate this stress. A high altitude environment can alter redox balance, and can induce oxidative stress. Oxidative damage to cellular DNA and proteins causes changes in cellular function (3). Acute and chronic hypoxic exposure at high altitude also leads to an impairment of the homeostatic regulation of Th1/Th2 immune balance (4). Accumulated reactive oxygen species (ROS) induced by altitude training can lead to oxidative stress damage, including apoptosis, lipid peroxidation, and DNA damage, and the occurrence of various diseases is precisely due to the long-term accumulation of peroxidation damage (5). During altitude training, the body receives dual stimulation by hypoxia and training, and the combination of the two stimuli increases and deepens the degree of oxidative stress damage. The exercise-induced immunosuppression and oxidative stress damage caused by altitude training can also induce a variety of diseases, which pose a significant threat to the health of athletes (6). Accelerating the adaptation process of athletes to altitude training so as to quickly improve the training effect is key to the success of altitude training. Some experts propose that hypoxic preconditioning before altitude training can decrease hypoxic stress, thereby reducing its impact on immune function and reducing infection.

Hypoxic preconditioning refers to a certain intensity and time of hypoxic exposure before hypoxic stress, which makes the body produce endogenous protection to enhance the body's tolerance to subsequent more severe hypoxia (7). Studies in animal models have shown that hypoxic preconditioning can reduce hypoxic damage and play a neuroprotective role in neonatal rats (8), and can significantly increase the exercise capacity of the rats in a hypoxic environment after hypoxic preconditioning (9). A clinical trial demonstrated that hypoxic preconditioning mobilizes endogenous protective mechanisms in the human body and facilitates an active adaptive response to oxidative stress, inflammatory responses, apoptosis, and excitotoxicity caused by ischemia and hypoxia (10). Furthermore, competitive sports studies have found that 2-week hypoxic preconditioning combined with 4-week altitude training can improve the oxygen carrying capacity of blood, and has a positive effect on the physical function status of swimmers (11). Additionally, 10-day hypoxic preconditioning combined with 8-week sub-altitude training was shown to improve the oxygen carrying capacity of blood and physical function status of rowers (12). However, there are few studies on the effects of hypoxic preconditioning combined with altitude training on the immune system of athletes.

The complement system is activated when pathogens enter the blood circulation. The system is not only an innate immune response of the host against pathogen invasion, but is also a fundamental part of adaptive and humoral immunity (13). Complement regulatory proteins can regulate the progression of the complement cascade at all levels to protect autologous cells (14). Decayaccelerating factor (CD55) and membrane inhibitor of reactive lysis (CD59) are 2 of the 4 major membranebinding complement regulatory proteins, and are widely expressed in all circulating cells and most human tissues. CD55 accelerates the degradation of invertase C3 and C5 and prevents the formation of new enzymes. CD59 interferes with the formation of the membrane attack complex (MAC) (15). CD55 and CD59, in addition to being complement inhibitors, are involved in the signaling of specific cellular receptors of the immune system or as ligands for these receptors. They participate in the activation and proliferation of lymphocytes and macrophages, and play important roles in regulating antigen presenting cells and regulating the secretion of cytokines and proinflammatory molecules (16).

This study investigated the changes in CD55 and CD59 expression on the surface of blood cells, as well as immune function in swimmers during hypoxic preconditioning combined with altitude training. The purpose was to further improve the current understanding of the effects of hypoxic preconditioning combined with altitude training on the complement regulation system and the immune function of athletes, so as to provide a reference and scientific basis for this mode of training. The results of this study are of great significance for better understanding the physical function status of athletes, the promotion of fatigue recovery, and monitoring immune levels during altitude training.

We present the following article in accordance with 
the MDAR reporting checklist (available at http://dx.doi. org/10.21037/apm-20-2379).

\section{Methods}

\section{Study subjects}

This study included 9 male swimmers from Shanghai. The mean age was $16.9 \pm 3.5$ years, mean height was $176.6 \pm 6.5$ $\mathrm{cm}$, mean weight was $74 \pm 5.3 \mathrm{~kg}$, and the mean training year was $5.8 \pm 2.2$. All procedures performed in this study involving human participants were in accordance with the Declaration of Helsinki (as revised in 2013).

\section{Training arrangements}

Living high-training low (HiLo) was performed for 3 weeks in the hypoxic laboratory of the Shanghai Research Institute of Sports Science. The initial simulated altitude was $1,500 \mathrm{~m}$ and increased to 2,200 m 3 days later until the end of the hypoxic preconditioning. The participants slept at the hypoxic laboratory from 19:00 pm to 6:00 am, 6 days a week, and underwent normal training in normoxic conditions during the day.

On the second day after the hypoxic preconditioning, they flew to Kunming (2,200 m above sea level) for 3-week altitude training. The training content and training intensity was consistent with the hypoxic preconditioning phase, and was led by the same coach.

Fasting venous blood was collected at 6:00 am for testing 4 times: before the start of hypoxic preconditioning, in the first week of hypoxic preconditioning, at the end of hypoxic preconditioning (i.e., before the start of altitude training), and at the end of altitude training.

Flow cytometry to detect the expression of CD 55 and CD59 on the surface of red blood cells (RBCs) and white blood cells (WBCs)

The blood samples were collected into EDTA tubes. Sample preparation was performed within 24 hours after blood collection. The number of RBCs and WBCs expressing CD55 and CD59 was determined by direct fluorescencelabeled flow cytometry. Mouse anti-human CD55-FITC monoclonal antibody $(10 \mu \mathrm{L}$, Beckman Coulter, America) and mouse anti-human CD59-PE monoclonal antibody (10 $\mu \mathrm{L}$, Beckman Coulter, America) were added into two numbered tubes, respectively. Then, $0.2 \mu \mathrm{L}$ EDTA blood sample was added to $50 \mu \mathrm{L}$ PBS solution, mixed thoroughly, and placed at room temperature in the dark for $30 \mathrm{~min}$. PBS solution was then added, centrifuged at 1,000 rpm for $5 \mathrm{~min}$, then the supernatant was aspirated. Finally, $300 \mu \mathrm{L}$ PBS solution was added and mixed for detection. CellQuest software (AMS Integrated, America) was used for data collection and analysis. Scatter diagrams with forward scatter and side scatter were used to distinguish blood cell populations. At least 10,000 cells were tested in each sample to analyze the relative expression of CD55 and CD59 on the surface of RBCs and WBCs.

\section{RBC count and WBC count}

A total of $1 \mathrm{~mL}$ EDTA blood sample and the Sysmex bloodcell counter were used for routine blood tests.

\section{Detection of immune system indexes $C D 3 \%, C D 4 \%$, and $C D 8 \%$}

CD3\%, CD4\%, and CD8\% were measured by Flow cytometry. Anticoagulant blood $(50 \mu \mathrm{L})$ was put into a clean tube, then $3 \mu \mathrm{L}$ mouse anti-human CD4+ monoclonal antibody labeled with RPE fluorescein and CD8+ monoclonal antibody labeled with FITC fluorescein were added and gently mixed. Anticoagulant blood $(50 \mu \mathrm{L})$ was put into another clean tube, and $3 \mu \mathrm{L}$ mouse anti-human CD4+ and CD8+ were added then gently mixed. After the two labeled tubes were incubated at room temperature in the dark for $15 \mathrm{~min}, 100 \mu \mathrm{L}$ hemolysin was added and gently mixed to destroy the RBCs. Then, $1 \mathrm{~mL}$ doubledistilled water was added and placed at room temperature in the dark for $5 \mathrm{~min}$. After centrifugation (1,200 rpm, $5 \mathrm{~min}$ ), the supernatant was aspirated and RBC debris were removed. After centrifuging again (1,200 rpm, $5 \mathrm{~min})$, the supernatant was collected. Finally, $0.5 \mathrm{~mL}$ PBS solution was added for detection. CellQuest software was used for detection and data analysis.

\section{Detection of immunoglobulins $\operatorname{Ig} G, \operatorname{Ig} M$, and $\operatorname{Ig} A$}

A total of $1 \mathrm{~mL}$ serum was used to detect immunoglobulins IgG, IgM, and IgA with the Hitachi 7100 automatic biochemical analyzer.

\section{Statistical analysis}

All data were analyzed using SPSS 19.0. After testing 
Table 1 Changes in decay-accelerating factor (CD55) and membrane inhibitor of reactive lysis (CD59) expression in red blood cells (RBCs) after hypoxic preconditioning combined with altitude training (mean fluorescence intensity)

\begin{tabular}{lcccc}
\hline Characteristics & $\begin{array}{c}\text { Before the start of hypoxic } \\
\text { preconditioning }\end{array}$ & $\begin{array}{c}\text { Hypoxic preconditioning } \\
\text { week 1 }\end{array}$ & $\begin{array}{c}\text { At the end of hypoxic preconditioning } \\
\text { (before the start of altitude training) }\end{array}$ & $\begin{array}{c}\text { At the end of altitude } \\
\text { training }\end{array}$ \\
\hline RBC count $\left(10^{12} / \mathrm{L}\right)$ & $5.01 \pm 0.23$ & $5.13 \pm 0.31$ & $5.25 \pm 0.34$ & $7.84 \pm 1.19^{\mathrm{b}}$ \\
CD55 in RBCs & $8.86 \pm 1.80$ & $15.39 \pm 3.31^{\mathrm{a}}$ & $-6.84 \%$ & $13.78 \pm 7.56^{\mathrm{c}}$ \\
Change rate & & $73.70 \%$ & $20.31 \pm 5.54^{\mathrm{b}}$ & $55.53 \%$ \\
CD59 in RBCs & $23.86 \pm 4.19$ & $46.94 \pm 9.49^{\mathrm{a}}$ & $-14.87 \%$ & $28.56 \pm 8.25^{\mathrm{bc}}$ \\
Change rate & & $96.73 \%$ & $19.70 \%$ \\
\hline
\end{tabular}

${ }^{\mathrm{a}}, \mathrm{P}<0.05$ vs. before the start of hypoxic preconditioning; ${ }^{\mathrm{b}}, \mathrm{P}<0.05$ vs. hypoxic preconditioning week $1 ;{ }^{\mathrm{c}}, \mathrm{P}<0.05$ vs. at the end of hypoxic preconditioning (before the start of altitude training).

normality, the indexes met the normal distribution. Repeated measures analysis of variance was used. All indexes all were expressed as mean $\pm \mathrm{SD}$, and $\mathrm{P}<0.05$ indicated a significant difference.

\section{Results}

\section{Effect of hypoxic preconditioning combined with altitude training on CD55 and CD59 expression in RBCs}

As shown in Table 1, CD55 expression in RBCs increased substantially after the start of hypoxic preconditioning and increased by $73.70 \%$ after 1-week of preconditioning, which was significantly higher than before the start of hypoxic preconditioning $(\mathrm{P}<0.05)$. Furthermore, CD55 expression decreased to baseline levels at the end of preconditioning, which was not significantly different from levels before the start of preconditioning. CD55 expression increased significantly during the subsequent altitude training, and was significantly higher than before altitude training $(\mathrm{P}<0.05)$. The changes in CD59 expression were similar to that of CD55. CD59 first increased then decreased in the preconditioning phase, and then increased again during the altitude training phase with significant differences compared to before altitude training. RBC count during the hypoxic preconditioning phase continued to increase, but there was no significant difference overall. RBC count during altitude training was significantly higher compared to before the start of preconditioning and in the first week of preconditioning $(\mathrm{P}<0.05)$.

\section{Effect of hypoxic preconditioning combined with altitude training on CD55 and CD59 expression in WBCs}

As shown in Table 2, CD55 expression in WBCs decreased significantly by $45.54 \%$ in the first week of hypoxic preconditioning $(\mathrm{P}<0.05)$, and remained at this decreased level at the end of hypoxic preconditioning. CD55 expression in WBCs increased substantially during altitude training, and was significantly higher than in the first week of hypoxic preconditioning and at the end of hypoxic preconditioning (before the start of altitude training) $(\mathrm{P}<0.05)$. CD59 expression in WBCs continued to decrease after the start of the hypoxic preconditioning phase, and reached minimum levels at the end of preconditioning, with a significant decrease of $58 \%$ compared to before the start of hypoxic preconditioning $(\mathrm{P}<0.05)$. CD59 expression also increased after the start of altitude training, and was significantly higher than that at the end of hypoxic preconditioning (before the start of altitude training). WBC count increased and then decreased during the hypoxic preconditioning phase. At the end of preconditioning, it was significantly lower than before preconditioning $(\mathrm{P}<0.05)$. WBC count increased again during altitude training, which was significantly higher than before preconditioning and before altitude training $(\mathrm{P}<0.05)$.

\section{Effects of hypoxic preconditioning combined with altitude training on the immune system}

As shown in Table 3, during hypoxic preconditioning, the changing trend of $\mathrm{CD} 3 \%$ was not significant. A comparison of each phase with the phase before preconditioning showed that CD3\% first decreased and then increased during hypoxic preconditioning, and decreased again at the end of altitude training, but there were no significant differences. During 3-week hypoxic preconditioning, CD4/ CD8 showed an increasing trend, and reached the highest point at the end of hypoxic preconditioning, which was 
Table 2 Changes in decay-accelerating factor (CD55) and membrane inhibitor of reactive lysis (CD59) expression in white blood cells (WBCs) after hypoxic preconditioning combined with altitude training (mean fluorescence intensity)

\begin{tabular}{lcccc}
\hline Characteristics & $\begin{array}{c}\text { Before the start of hypoxic } \\
\text { preconditioning }\end{array}$ & $\begin{array}{c}\text { Hypoxic preconditioning } \\
\text { week } 1\end{array}$ & $\begin{array}{c}\text { At the end of hypoxic preconditioning } \\
\text { (before the start of altitude training) }\end{array}$ & $\begin{array}{c}\text { At the end of altitude } \\
\text { training }\end{array}$ \\
\hline WBC count $\left(10^{9} / \mathrm{L}\right)$ & $6.29 \pm 1.36$ & $6.76 \pm 1.29$ & $5.26 \pm 1.54^{\mathrm{a}}$ & $7.05 \pm 1.61^{\mathrm{ac}}$ \\
CD55 in WBCs & $18.84 \pm 4.99$ & $10.26 \pm 2.52^{\mathrm{a}}$ & $10.60 \pm 6.64$ & $-43.73 \%$ \\
Change rate & - & $-45.54 \%$ & $2.31 \pm 0.36^{\mathrm{a}}$ & $-10.46 \%$ \\
CD59 in WBCs & $5.50 \pm 3.26$ & $2.67 \pm 1.41$ & $-58.00 \%$ & $3.30 \pm 0.36^{\mathrm{c}}$ \\
Change rate & - & $-51.45 \%$ & $-40.00 \%$ & \\
\hline
\end{tabular}

${ }^{a}, \mathrm{P}<0.05$ vs. before the start of hypoxic preconditioning; ${ }^{b}, \mathrm{P}<0.05$ vs. hypoxic preconditioning week $1 ;{ }^{\mathrm{c}}, \mathrm{P}<0.05$ vs. at the end of hypoxic preconditioning (before the start of altitude training).

Table 3 Changes in T lymphocytes and their subsets (CD3\%, CD4\%, CD8\%, and CD4/CD8) and immunoglobulins (IgG, IgM, and IgA) during hypoxic preconditioning combined with altitude training

\begin{tabular}{|c|c|c|c|c|}
\hline Characteristics & $\begin{array}{l}\text { Before the start of } \\
\text { hypoxic preconditioning }\end{array}$ & Hypoxic preconditioning week 1 & $\begin{array}{l}\text { At the end of hypoxic preconditioning } \\
\text { (before the start of altitude training) }\end{array}$ & $\begin{array}{l}\text { At the end of } \\
\text { altitude training }\end{array}$ \\
\hline CD3\% & $65.47 \pm 5.18$ & $63.21 \pm 4.37$ & $66.46 \pm 5.83$ & $65.62 \pm 4.89$ \\
\hline CD8\% & $30.01 \pm 4.14$ & $28.34 \pm 3.14$ & $28.22 \pm 4.05$ & $29.56 \pm 3.92$ \\
\hline CD4/CD8 & $1.35 \pm 0.34$ & $1.38 \pm 0.32$ & $1.45 \pm 0.36^{\mathrm{a}}$ & $1.21 \pm 0.39^{\mathrm{ac}}$ \\
\hline $\lg A(g / L)$ & $1.62 \pm 0.45$ & $1.57 \pm 0.67$ & $1.55 \pm 0.34$ & $1.58 \pm 0.52$ \\
\hline $\lg M(g / L)$ & $0.92 \pm 0.31$ & $1.13 \pm 0.45$ & $0.95 \pm 0.46$ & $0.97 \pm 0.44$ \\
\hline
\end{tabular}

${ }^{a}, \mathrm{P}<0.05$ vs. before the start of hypoxic preconditioning; ${ }^{\circ}, \mathrm{P}<0.05$ vs. at the end of hypoxic preconditioning (before the start of altitude training).

significantly different from that before preconditioning $(\mathrm{P}<0.05)$. It began to decrease during altitude training and was significantly different from before altitude training $(\mathrm{P}<0.05)$.

In the hypoxic preconditioning phase, IgG and $\operatorname{IgM}$ increased and then decreased, while IgA continued to decrease, with no significant difference in each phase of preconditioning. In the altitude training phase, all three immunoglobulins increased slightly, but there was no significant difference in each phase of training.

\section{Discussion}

The expression of CD55 and CD59 on the RBC surface after hypoxic preconditioning increases significantly, which is a compensatory effect of the body on changes in the environment. A hypoxic environment induces immune cell activation and affects immune function (17). CD55 and CD59 expression on the RBC surface were significantly increased in swimmers during the early phase of 2-week HiLo training and rowers during the early phase of 3 -week HiLo training, indicating that CD55 plays a key role in preventing complement activation and preventing complement attack on RBCs $(18,19)$. In this study, after 1-week of hypoxic preconditioning, CD55 and CD59 expression were higher than before training. Increased CD55 expression is beneficial for the maintenance of RBC stability and prevents the occurrence of cell hemolysis. After 1-week of hypoxic preconditioning, CD55 and CD59 expression in RBCs decreased significantly until the end of hypoxic preconditioning. The decreased expression of CD55 and CD59 proteins on RBC membranes may be due to impaired synthesis of GPI-anchors or abnormal coupling of the membranes and proteins on RBC precursor 
membranes (20). Complement system disorder is partially responsible for increased hemolysis and tissue damage (21).

The significant increase in CD55 and CD59 expression in RBCs during altitude training is an adaptive adjustment of the body to re-hypoxic exposure. The adjustment is effective for maintaining the function of RBCs and reducing complement attack on RBCs. Hypoxic preconditioning combined with altitude training can increase the expression of CD55 and CD59, which is beneficial for resisting complement attack.

During 3-week hypoxic preconditioning, RBC count increased slightly, but there was no significant difference, suggesting that the expression of CD55 and CD59 on the RBC surface was not related to RBC count, but only related to the regulation of the complement system. During the following 3-week altitude training, RBC count increased substantially, and was significantly higher than before hypoxic preconditioning. Gao et al. (11) found that in a study of swimmers who underwent 2-week hypoxic preconditioning combined with 4-week altitude training, RBC count change did not change significantly during the hypoxic preconditioning phase. This result was consistent with our findings. They also found that RBC count reached the maximum value in the third week of altitude training, which was significantly higher than before hypoxia and in the first week of hypoxia, and decreased in the fourth week. Different times and modes of hypoxic training have different effects on RBCs, which may be related to the different height and intensity of hypoxic training.

The WBC count first increased and then decreased during hypoxic preconditioning, which was consistent with the results of Gao et al. (22) who examined changes in WBCs during HiLo training of rowers. The increase in WBC count at the early phase of preconditioning was likely to be a stress phenomenon of the body to the hypoxic environment, while the subsequent decrease in WBC count might be due to the deepening of hypoxic stimulation and the training load, resulting in greater impact on the body. Wang et al. (23) found that in a study of boxers who underwent 4-week HiLo, WBC count decreased in the first week, and then increased until the end of HiLo, with no significant difference at each phase. This result is different from our findings. In our study, the WBC count decreased significantly at the end of hypoxic preconditioning, which might be related to different training load and time. Most studies have found that the WBC levels of athletes in different events decreases significantly during subaltitude training and altitude training (24). In this study, during altitude training, WBCs significantly increased and immunosuppression was weakened in swimmers, suggesting that after 3 -week hypoxic preconditioning, the body adapted well to altitude training, and hypoxic preconditioning improved the immunity of the athletes. At the same time, this study also found that the changes in CD55 and CD59 expression on the WBC surface was not significantly related to $\mathrm{WBC}$ count, which is consistent with the results of Zhao et al. (25) who demonstrated that that the expression of CD55 and CD59 on the WBC surface is related to the complement regulatory system itself and not the WBC count.

The function of CD55 and CD59 is not limited to the innate immune system by regulating the complement system. It has been shown that CD55, CD59, and other complement regulators are able to reduce $\mathrm{T}$ cell responses and proliferation (26), which may explain the expression of CD55 and CD59 in WBCs and its subtypes. The expression of CD55 and CD59 varies greatly in different cell lines, while CD59 is more highly expressed on RBCs (27), which is also consistent with the results of this study. In this study, the expression of CD59 in RBCs was significantly higher than in WBCs. It has been reported that the regulation of CD55 and CD59 by exposure to nucleated cells, proinflammatory molecules, and cytokines (e.g., C-reactive protein, TNF- $\alpha, \mathrm{IL}-1)$ is an adaptive response to a chronic inflammatory state (28), associated with long-term systemic complex activation (29). However, the biological functions of these molecules are related to the cell membranes which express CD55 and CD59. RBC membrane proteomes, unlike WBCs (as well as other nucleated cells), are rarely altered but remain constant in the activated state of the cells (30). This may be why the expression of CD55 and CD59 on the RBC surface is not the same as that shown in this study.

Artificial simulated hypoxic training had no significant effect on T lymphocytes in athletes. Gao et al. (31) found that in a study of the immune function of female rowers who underwent 4-week HiLo, CD4 and CD4/CD8 increased significantly after the start of HiLo until the end of training, while CD8 did not change significantly during the whole process. This result is similar to our findings. In this study, CD4/CD8 was significantly increased during 3 -week hypoxic preconditioning, suggesting that hypoxic preconditioning in HiLo mode improved the immune function of the athletes. During the subsequent altitude training, CD4/CD8 continued to decrease, indicating that the altitude training model inhibited cellular immunity. At the end of the altitude training, although $\mathrm{CD} 4 / \mathrm{CD} 8$ was 
significantly lower than before altitude training, it was not significantly different from that before the start of hypoxic preconditioning. This indicates that altitude training after hypoxic preconditioning inhibited $\mathrm{T}$ lymphocyte immunity, but did not have a significant effect on immune function, only on immune balance. The reasons behind this are likely related to the increase of RBCs and hemoglobin during hypoxic preconditioning, which improved the oxygen carrying ability of blood and the aerobic capacity of muscles, which in turn improves immunity.

CD3 first decreased and then increased during the hypoxic preconditioning phase, but there was no significant difference, suggesting that the hypoxic preconditioning phase had little effect on CD3. This result is similar to the previous finding that CD3 does not change significantly after 3-week HiLo in rowers. Xu et al. (32) found that by comparing four different hypoxic preconditioning modes, the hypoxic preconditioning mode of HiLo had little effect on T lymphocyte immunity. Chang et al. (33) found that in a study of 3 sub-altitude training modes on the Chinese Short Track Speed Skating Team, CD3 decreased significantly after the end of 3 -week altitude training, indicating that altitude training greatly stimulated CD3. In this study, although CD3 decreased slightly at the end of altitude training after 3 -week hypoxic preconditioning, there was no significant difference compared with that before hypoxic preconditioning and before altitude training, indicating that hypoxic preconditioning caused an adaptive immune response.

There are few studies on the effects of altitude training on immunoglobulins. Wang et al. (34) found that the levels of the three immunoglobulins in rowers did not change significantly during 8 -week sub-altitude training $(1,400 \mathrm{~m})$, and IgM showed a significant increase 2 weeks after leaving the altitude environment. Furthermore, Gao et al. (35) found that the levels of the 3 immunoglobulins in rowers were basically stable without significant changes during 8 -week altitude training $(2,800 \mathrm{~m})$. In this study, we found that although the trends of $\operatorname{IgG}, \operatorname{IgM}$, and $\operatorname{IgA}$ were different during hypoxic preconditioning, the overall changes were not significant. At the end of hypoxic preconditioning, the levels of all three immunoglobulins tended to decrease, indicating that hypoxic preconditioning inhibited cellular immunity. The small increase in the three immunoglobulins during altitude training suggested that the cellular immune response tended to improve. However, considering that the range of change of the immunoglobulins was not large, and there was no significant difference in each phase of hypoxic preconditioning combined with altitude training, it is possible that hypoxic preconditioning and altitude training had little effect on cellular immunity.

\section{Conclusions}

Our study demonstrated that during hypoxic preconditioning, the complement regulation system and RBC immunity were inhibited, and T lymphocyte immunity was out of balance. After hypoxic preconditioning combined with altitude training, the expression of CD55 and CD59 on the surface of RBCs and WBCs increased significantly, and $\mathrm{T}$ lymphocyte CD4/CD8 increased. These results suggest an improvement of the complement regulation system and RBC immune function after hypoxic preconditioning. Hypoxic preconditioning can improve immune cell levels, and therefore and have a beneficial effect on the physical function of athletes during altitude training.

It is suggested that appropriate hypoxic preconditioning be performed before altitude training in the future, which can improve the body's immune function and reduce the risk of disease during altitude training.

\section{Acknowledgments}

Funding: This study was supported by the Shanghai Science and Technology Committee (19dz1200700).

\section{Footnote}

Reporting Checklist: The authors have completed the MDAR reporting checklist. Available at http://dx.doi.org/10.21037/ apm-20-2379

Data Sharing Statement: Available at http://dx.doi. org/10.21037/apm-20-2379

Conflicts of Interest: All authors have completed the ICMJE uniform disclosure form (available at http://dx.doi. org/10.21037/apm-20-2379). The authors have no conflicts of interest to declare.

Ethical Statement: The authors are accountable for all aspects of the work in ensuring that questions related to the accuracy or integrity of any part of the work are appropriately investigated and resolved. All procedures performed in this study involving human participants were in accordance with the Declaration of Helsinki (as revised in 2013). 
Open Access Statement: This is an Open Access article distributed in accordance with the Creative Commons Attribution-NonCommercial-NoDerivs 4.0 International License (CC BY-NC-ND 4.0), which permits the noncommercial replication and distribution of the article with the strict proviso that no changes or edits are made and the original work is properly cited (including links to both the formal publication through the relevant DOI and the license). See: https://creativecommons.org/licenses/by-nc-nd/4.0/.

\section{References}

1. Gough CE, Saunders PU, Fowlie J, et al. Influence of altitude training modality on performance and total haemoglobin mass in elite swimmers. Eur J Appl Physiol 2012;112:3275-85.

2. Rodríguez FA, Iglesias X, Feriche B, et al. Altitude Training in Elite Swimmers for Sea Level Performance (Altitude Project). Med Sci Sports Exerc 2015,47:1965-78.

3. Bakonyi T, Radak Z. High altitude and free radicals. J Sports Sci Med 2004;3:64-9.

4. Facco M, Zilli C, Siviero M, et al. Modulation of immune response by the acute and chronic exposure to high altitude. Med Sci Sports Exerc 2005;37:768-74.

5. Fatouros IG, Destouni A, Margonis K, et al. Cell-free plasma DNA as a novel marker of aseptic inflammation severity related to exercise overtraining. Clin Chem 2006;52:1820-4.

6. Dong JM, Wang JB, Qin LL, et al. Research advances on biomarkers of immune responses associated with exercise induced oxidative Stress in plateau environment. Journal of Environmental \& Occupational Medicine 2015,32:486-90

7. Huang L, Wu S, Li H, et al. Hypoxic preconditioning relieved ischemic cerebral injury by promoting immunomodulation and microglia polarization after middle cerebral artery occlusion in rats. Brain Res 2019;1723:146388.

8. Parmar J, Jones NM. Hypoxic preconditioning can reduce injury-induced inflammatory processes in the neonatal rat brain. Int J Dev Neurosci 2015;43:35-42.

9. Wang LJ, Wei H, Yan L, et al. Effects of hypoxic preconditioning on Nrf2 and mitochondril respiratory chain complex protein expression and exercise ability in skeletal muscle of mice. Journal of Beijing Sport University 2019,42:126-32.

10. Baillieul S, Chacaroun S, Doutreleau S, et al. 2017. Hypoxic conditioning and the central nervous system: a new therapeutic opportunity for brain and spinal cord injuries? Exp Biol Med (Maywood) 2017;242:1198-206.

11. Gao H, Gao BH. Effect of hypoxic pre-conditioning and altitude training on physical functions of swimmers. China Sport Science and Technology 2009;45:62-5.

12. Wang G, Gao BH, Gao H. Effect of hypoxic preconditioning and long time sub-altitude training on athletic ability of rowers. Journal of Xi'an Physical Education University 2013,30:327-33.

13. Dunkelberger JR, Song WC. Complement and its role in innate and adaptive immune responses. Cell Res 2010;20:34-50.

14. Zipfel PF, Skerka C. Complement regulators and inhibitory proteins. Nat Rev Immunol 2009;9:729-40.

15. Walport MJ. Complement. Second of two parts. N Engl J Med 2001;344:1140-4.

16. Asimakopoulos JV, Terpos E, Papageorgiou L, et al. The presence of CD55- and/or CD59-deficient erythrocytic populations in patients with rheumatic diseases reflects an immunemediated bone-marrow derived phenomenon. Med Sci Monit 2014;20:123-39.

17. Chen RY, Meng M, Zhang J, et al. Effects of exposure to simulate unpressurised and dry state of disable submarimne on the distributions of CD55 and CD59 on blood cellular membrane. Chinese Journal of Applied Physiology 2007;23:226-8.

18. Zhao YC, Gao BH, Ding SZ. Effect of HiLo on the Expression of Immune Molecules and Immune Function in the Erythrocyte of Swimmers. China Sport Science 2010;30:66-71.

19. Wang X, Gao BH. Increased CD55 and CD59 expressions of elite male rowers after 3-week hypoxic training. Chinese Journal of Sports Medicine 2014;33:1054-9.

20. Richaud-Patin Y, Perez-Romano B, Carrillo-Maravilla E, et al. Deficiency of red cell bound CD55 and CD59 in patients with systemic lupus erythematosus patients. Immunol Lett 2003,88:95-9.

21. Ubol S, Masrinoul P, Chaijaruwanich J, et al. Differences in global gene expression in peripheral blood mononuclear cells indicate a significant role of the innate responses in progression of dengue fever but not dengue hemorrhagic fever. J Infect Dis 2008;197:1459-67.

22. Gao BH, Gao H, Chen J, et al. Different Model of Hypoxia Training on White Blood Cell Count of Female Rowers. Journal of Tianjin University of Sport 2006;(1):27-30.

23. Wang YY, Li WJ, Gao YQ, et al. The Effect of Living High and Training Low of Simulation on the Immune 
Index of the Boxing Youth Athletes. China Sport Science and Technology 2019;55:37-42.

24. Yu T, Chang Y, Zhao P, et al. Effects of Sub-Altitude Training on the Physiological Functions of the Elite Female Weightlifters. China Sport Science 2016;36:67-71.

25. Zhao Y, Yu DH, Yang HX. Long-term Taijiquan Practice Antagonizes Effectively the Decrease in Immune Function of Aging Women. Chinese Journal of Sports Medicine 2016;35:417-22.

26. Kemper C, Atkinson JP. T-cell regulation: with complements from innate immunity. Nat Rev Immunol 2007;7:9-18.

27. Hernandez-Campo PM, Almeida J, Sanchez ML, et al. Normal patterns of expression of glycosylphosphatidylinositol- anchored proteins on different subsets of peripheral blood cells: A frame of reference for the diagnosis of paroxysmal nocturnal hemoglobinuria. Cytometry B Clin Cytom 2006;70:71-81.

28. Li SH, Szmitko PE, Weisel RD, et al. C-reactive protein upregulates complement-inhibitory factors in endothelial cells. Circulation 2004;109:833-6.

29. van Beek J, van Meurs M, 't Hart BA, et al. Decayaccelerating factor (CD55) is expressed by neurons in response to chronic but not acute autoimmune central nervous system inflammation associated with complement activation. J Immunol 2005;174:2353-65.

Cite this article as: Wang X, Huang L, Gao H. Effects of hypoxic preconditioning combined with altitude training on CD55, CD59 and the immune function of swimmers. Ann Palliat Med 2021;10(1):509-517. doi: 10.21037/apm-20-2379
30. García-Valladares I, Atisha-Fregoso Y, Richaud-Patin Y, et al. Diminished expression of complement regulatory proteins (CD55 and CD59) in lymphocytes from systemic lupus erythematosus patients with lymphopenia. Lupus 2006;15:600-5.

31. Gao BH, Chen PJ. Effects of 4-weeks Living LowTraining High on the Immune Function of Chinese Female Rowers. Chinese Journal of Sports Medicine 2014;33:404-12.

32. Xu GQ, Lin WT, Wen XQ, et al. The Effects of Different Hypoxic Preconditioning Methods on the Leucocyte Subpopulations of SD Rats. China Sport Science 2006;(11):84-7.

33. Chang Y, He ZH, Wang LR, et al. Effects of medium-level altitude training on the cell immune function of elite speed skaters. China Sport Science 2002,22:86-90.

34. Wang G, Gao BH, Gao H. Body function of rowers before and after hypoxic pre-conditioning and chronic sub-altitude training. Chinese Journal of Sports Medicine 2013;32:489-94.

35. Gao H, Gao BH, Feng LS, et al. Effect of Long-term Altitude Training on the Immunity and Plasma Leptin of Elite Rowers. Chinese Journal of Sports Medicine 2011;30:986-91.

(English Language Editor: C. Betlazar-Maseh) 